\title{
The Value of Continuous Noninvasive Hemoglobin Monitoring in Intraoperative Blood Transfusion Practice during Abdominal Cancer Surgery
}

\author{
Ahmed Mostafa Kamal1* ${ }^{*}$, Mohamed Adly Elramely², Mohamed Mohamed Abd Elhaq ${ }^{1}$ \\ ${ }^{1}$ Faculty of Medicine, Cairo University, Giza, Egypt \\ ${ }^{2}$ National Cancer Institute, Cairo University, Giza, Egypt \\ Email: ‘ahmedmostafakamal@yahoo.com,mramely@hotmail.com,mohamedabdelhaq76@hotmail.com
}

Received 19 February 2016; accepted 14 March 2016; published 17 March 2016

Copyright (C) 2016 by authors and Scientific Research Publishing Inc.

This work is licensed under the Creative Commons Attribution International License (CC BY).

http://creativecommons.org/licenses/by/4.0/

(c) (i) Open Access

\section{Abstract}

Introduction: Patients undergoing major oncological surgery may suffer from severe bleeding. Sometimes, it is difficult to anesthesiologist to take decision about timing of administration blood products to such patients. The aim of this study is to evaluate the use of continuous noninvasive hemoglobin monitoring as a guide for blood transfusion practice. Methods: One hundred patients undergoing elective abdominal cancer surgeries were randomly allocated into two groups, Group I $(n=50)$ : laboratory $\mathrm{Hb}$ was obtained at baseline (immediate preoperative), intraoperative (when to suggest transfusion triggering value) and immediate postoperative. Group II $(n=50)$ : The probe of Masimo for $\mathrm{SpHb}$ monitoring was applied immediately after induction of anesthesia at the index finger. Laboratory $\mathrm{Hb}$ was obtained at baseline (immediate preoperative), intraoperative (when to suggest transfusion triggering value) and immediate postoperative. Results: A number of transfused units of RBC were significantly lower in SpHb group than in control group (p value $<0.05$ ), and a number of saved $R B C$ units were significantly higher in SpHb group than in control group (p value $<\mathbf{0 . 0 0 1}$ ). The correlation between $\mathrm{Lab} \mathrm{Hb}$ and $\mathrm{SpHb}$ was highly significant between baseline Lab $\mathrm{Hb}$ and baseline $\mathrm{SpHb}(\mathrm{r}=0.698, \mathrm{p}<0.001)$. Similarly, Lab $\mathrm{Hb}$ before transfusion showed a significant correlation between SpHb before transfusion $(r=0.710, p<0.001)$. On the contrary, there was a non-significant correlation between Lab $\mathrm{Hb}$ after transfusion and $\mathrm{SpHb}$ after transfusion $(r=0.045, p>0.05)$. Conclusion: SpHb monitoring had clinically acceptable absolute and trend accuracy. SpHb monitoring altered transfusion decision making and resulted in decreased RBC utilization and decreased RBC costs while facilitating earlier transfusions when indicated.

\footnotetext{
${ }^{*}$ Corresponding author.
}

How to cite this paper: Kamal, A.M., Elramely, M.A. and Abd Elhaq, M.M. (2016) The Value of Continuous Noninvasive Hemoglobin Monitoring in Intraoperative Blood Transfusion Practice during Abdominal Cancer Surgery. Open Journal of Anesthesiology, 6, 13-19. http://dx.doi.org/10.4236/ojanes.2016.63003 


\section{Keywords}

\section{Masimo, Intraoperative, Blood Transfusion, Abdominal Cancer Surgery}

\section{Introduction}

Major oncological surgery patients are at risk of severe bleeding due to the tumor biology, pre-operative cancer therapies (chemo-radiation and immune-therapies), and perioperative factors e.g. hemodilution, and hypothermia. A recent report shows that the rate of perioperative blood transfusions (BTs) in colorectal and prostate cancer patients has decreased from $68.6 \%$ to $45.6 \%$ over the last three decades. However, more effort is needed to reduce this rate [1]. Sometimes, it is difficult to anesthesiologist to take decision about timing of administration blood products to patients undergoing cancer surgery, especially there are no clear indications or consensus guidelines exist. Moreover, a decreased immune surveillance as a consequence of blood transfusions has been linked to cancer recurrence and progression [2].

Anemia is common amongst surgical patients and independently associated with adverse outcomes [3]. A recent systematic analysis by an expert panel of 494 studies evaluating RBC transfusions for actual benefit in health outcomes concluded that 59\% of transfusions were, in fact, inappropriate [4]. Accordingly, benefits of RBC transfusion to mitigate anemia should outweigh the risks associated with blood products administration. However, laboratory hemoglobin $(\mathrm{Hb})$ is the primary indicator of the need for blood transfusion [5]. It is only intermittently available and results can be delayed. Apparently, during surgery, initial and subsequent transfusion decisions are often made without recent $\mathrm{Hb}$ results [6]. Consequently, a reliable, real-time monitor is needed to guide blood and fluid resuscitation [7].

The appropriate target range for hemoglobin during surgery is likely patient-specific, but most patients will tolerate hemoglobin in the range of $7-9 \mathrm{~g} / \mathrm{dl}$. Maintaining hemoglobin within a target range during surgery by titrated transfusion could limit the potentially adverse effects of anemia and transfusion related complications [8].

Currently, transfusion decisions are guided by visual estimation of surgical blood loss with periodic hemoglobin measurement using invasive means, such as clinical laboratory testing, or arterial blood gas for determination of hemoglobin level (arterial hemoglobin) [9].

Continuous and noninvasive hemoglobin $(\mathrm{SpHb})$ monitoring is now possible with Pulse CO-Oximetry and multi-wavelength sensors, which also provide traditional pulse Oximetry measurements [10]. SpHb is available as a part of the Masimo rainbow ${ }^{\circledR}$ SET platform [11]. Rainbow SET and Signal Extraction Technology (SET) are based on similar principles. Light is passed through tissue and light that passes entirely through is measured. The difference between the amount that is sent through the tissue (emitted) and the amount that emerges (detected) equals the amount of light that is absorbed by the tissue bed, capillaries, arteries, and veins. The absorbed amount is then compared to the known absorption spectra of a particular blood constituent-in this case hemoglobin [11].

The aim of this study is to evaluate the use of continuous noninvasive intraoperative hemoglobin monitoring using Masimo Radical-7 ${ }^{\mathrm{TM}}$ Pulse CO-Oximeter during elective abdominal cancer surgeries as a guide for blood transfusion practice.

\section{Patients and Methods}

This double-blinded randomized controlled clinical trial was carried out in Cairo University hospitals, between June 2014 and October 2015, after obtaining approval of the departmental scientific and ethical committee. One hundred ASA physical statuses I, II patients of both sexes, 18 - 65 years old, hundred patients undergoing elective abdominal cancer surgeries were included in the study after obtaining written informed consent from all patients enrolled in our study. Patient were excluded from study in case of a known cardiac arrhythmias, intracardiac shunts, left or right ventricular dysfunction, peripheral circulation failure of the upper extremities, chronic obstructive pulmonary disease, significant liver disease, significant renal disease, pregnancy, coagulopathy, the use of antiplatelet or anticoagulants and $\mathrm{Hb} \%$ less than $10 \mathrm{gm} / \mathrm{dl}$ were be excluded from the study.

Patients were randomly allocated into two equal groups. The randomization sequence was concealed in opa- 
que sealed envelopes that were kept by the senior anesthesia staff. The envelope was opened at the beginning of the operation. Patients were randomly allocated to either:

Group I (n = 50): Control group, Laboratory $\mathrm{Hb}$ will be obtained at baseline (immediate preoperative), intraoperative (when to suggest transfusion triggering value) and immediate postoperative.

Group II (n = 50): Masimo group, The Probe of Masimo for SpHb monitoring (Radical-7 ${ }^{\mathrm{TM}}$ Pulse CO-Oximeter and Rainbow deposable ${ }^{\mathrm{TM}}$ Adhesive Sensor, Revision E, Masimo, Irvine, CA) was be applied immediately after induction of anesthesia at the index finger. Laboratory $\mathrm{Hb}$ was also obtained at baseline (immediate preoperative), intraoperative (when to suggest transfusion triggering value) and immediate postoperative.

Primary outcome of this study was to evaluate reliability of Massimo probe for decision of initiation and cessation of blood transfusion for patients undergoing elective abdominal cancer surgeries. But the transfusion delay time and haemodynamic changes intra operatively were considered secondary outcome.

All patients were subject to systematic preoperative assessment including history taking, physical examination, and review of the results of routine investigations, on arrival to operative room, electrocardiogram, pulse oximetry, capnography, Core temperature and non-invasive arterial blood pressure at 5 minutes intervals were applied.

General anesthesia was induced in all patients in supine position with i.v. Propofol, fentanyl, Atracurium, oral cuffed endotracheal tube, anesthesia was maintained with oxygen $60 \%$, air $40 \%$, sevoflurane, additional doses of Atracurium, mechanical ventilation with the maintenance of end-tidal carbon dioxide $35-40 \mathrm{mmHg}$. After induction and endotracheal intubation large bore venous access, insertion of indwelling urinary catheter was done, and a radial arterial catheter was placed for blood pressure monitoring and arterial hemoglobin analysis.

Patients in the Control Group received intraoperative $\mathrm{Hb}$ monitoring by intermittent blood sampling analyzed by the central laboratory. An initial blood sample was taken prior to surgery (baseline $\mathrm{Hb}$ ) in all patients. Intra operatively, blood samples were taken when estimated blood lossaround $15 \%$ of total estimated blood volume. In $\mathrm{SpHb}$ Group, patients were monitored with a Radical-7 Pulse CO-Oximeter, v7748, connected to a R2-25 adult ReSposable sensor (Revision E, Masimo, Irvine, CA) that displayed continuous SpHb values. After induction of anesthesia, the sensor was placed on the ring finger of the non-dominant hand contralateral to the arterial line. In both groups transfusion was started when $\mathrm{Hb}$ was $<10 \mathrm{~g} / \mathrm{dL}$, $\mathrm{Hb}$ value was confirmed to be $10 \mathrm{~g} / \mathrm{d}$ Blood sampling technique was the same for both groups. Blood loss for each patient was estimated by the number of saturated sponges or surgical gauze, the amount of suctioned blood in the waste canister, and the amount of blood in the surgical field. At the end of surgery, inhalational anesthetic was discontinued. Neuromuscular blockade was reversed with neostigmine and atropine IV. After that, extubation was done on fully awake patients. All patients were transferred to post anesthetic care unite (PACU) where they were monitored.

\section{Statistical Analysis}

According to Wael et al. [12] who assumed that patients necessitating a blood transfusion would require an average of 1.8 units of blood. Therefore, in order to detect a 33\% reduction in red blood cell units transfused with the addition of $\mathrm{SpHb}$ monitoring (expected effect size equal to 0.6 units) with a standard deviation of 1.0 unit [12], and two-tailed $\alpha$ error probability of 0.05 and power of $85 \%$; 45 subjects would be required for each group.

All the statistical analyzes were performed using The SPSS software (version 14.0; SPSS Inc, Chicago, IL, USA). Data presented as a mean and standard deviation or number and percentage. For evaluation of differences between groups, two pro portion Z-tests, two sided t-tests or Mann-Whitney rank sum tests for non-normally distributed data were performed, as appropriate, with a p value $<0.05$ considered statistically significant.

\section{Results}

One hundred patients who fulfilled the inclusion criteria, were enrolled in the study, all enrolled patients have already completed the study. Those patients were randomly allocated into two groups, 50 patients each. Patient demographic data showed no significant differences as regard age, gender, ASA, type of surgery, and duration of operation in both studied groups (Table 1).

Transfusion variables as regard blood loss, the percentage of patients transfused, patients with blood loss more than $15 \%$ in each group were not statistically significant difference between both studied group (p value > 0.05). But number of transfused units of RBC were significantly lower in SpHb group than in control group (p value 0.02), and number of saved RBC units were significantly higher in SpHb group than in control group (p value $<0.001$ ) (Table 2 ). 
Table 1. Demographic data.

\begin{tabular}{lll}
\hline & Group I n (50) & Group II n (50) \\
\hline Age & $39.85 \pm 13.34$ & $36.93 \pm 15.04$ \\
Gender & Males: 19 (38\%) & Males: 25 (50\%) \\
${ }^{\dagger}$ ASA & Females: $31(62 \%)$ & Females: 25 (50\%) \\
I & & \\
II & $16(32 \%)$ & $21(42 \%)$ \\
Type of surgery & $34(68 \%)$ & $24(48 \%)$ \\
- Cancer stomach & & \\
- Wipple surgery & $12(24 \%)$ & $10(20 \%)$ \\
- Cancer colon & $10(20 \%)$ & $8(16 \%)$ \\
- Hepatic resection & $23(46 \%)$ & $25(50 \%)$ \\
Duration of operation in hours & $5(10 \%)$ & $7(14 \%)$ \\
\hline
\end{tabular}

Data are described as mean \pm SD or number of patients (percentage \%), ${ }^{\dagger}$ ASA American Society of Anesthesiologists.

Table 2. Transfusion data.

\begin{tabular}{cccc}
\hline & Group I & Group II & P \\
\hline Blood loss (ml) & $1.750 \pm 655$ & $1.690 \pm 825$ & 0.28 \\
Transfused patients & $30(60 \%)$ & $32(64 \%)$ & 0.12 \\
Patients with blood loss exceeded 15\% & $15(30 \%)$ & $17(34 \%)$ & 0.31 \\
No of transfused units & $3.97 \pm 1.64$ & $2.42 \pm 1.38$ & 0.02 \\
No of saved units & $0.37 \pm 0.55$ & $1.55 \pm 0.90$ & $<0.001$ \\
\hline
\end{tabular}

Data are described as mean \pm SD or number of patients (percentage \%), P value $<0.05$ is considered statistically significant.

About base line hemoglobin and pre transfusion hemoglobin; there were no significant difference between both study groups, but post transfusion hemoglobin was significantly lower in SpHb group (10.3 \pm 0.4$)$ than control group $(10.9 \pm 0.7) \mathrm{p}$ value $<0.05$. The lag time before transfusion was statistically significant lower in SpHb group $(10.5 \pm 2.3)$ than in control group $(43.5 \pm 8.6)$ p value $<0.001$ (Table 3$)$.

By using Pearson correlation coefficient to detect the correlation between Lab $\mathrm{Hb}$ and $\mathrm{SpHb}$, there was a highly significant correlation between baseline Lab Hb and baseline SpHb $(r=0.698, \mathrm{p}<0.001)$. Similarly, Lab $\mathrm{Hb}$ before transfusion showed a significant correlation between with SpHb before transfusion $(\mathrm{r}=0.710, \mathrm{p}<$ 0.001). On the contrary, there was a non-significant correlation between Lab $\mathrm{Hb}$ after transfusion and $\mathrm{SpHb}$ after transfusion $(r=0.045, \mathrm{p}>0.05)$ (Figure 1$)$.

\section{Discussion}

This study was performed to evaluate efficacy of noninvasive hemoglobin monitoring in decision of initiation and cessation of blood transfusion in patient undergoing elective abdominal cancer surgeries. The result of this study showed that a number of transfused units of RBC were significantly lower in $\mathrm{SpHb}$ group than in control group (p value 0.02), and number of saved RBC units were significantly higher in SpHb group than in control group ( $p$ value $<0.001$ ). Also the result revealed that, base line hemoglobin and pre transfusion hemoglobin level were not statistically significant difference between both studied groups but post transfusion hemoglobin was significantly lower in SpHb group $(10.3 \pm 0.4)$ than in control group $(10.9 \pm 0.7)$ p value $<0.05$. The lag time before transfusion was statistically significant lower in SpHb group (10.5 \pm 2.3$)$ than in control group (43.5 \pm 8.6) p value $<0.001$. The result of our study correlates well with Ehrenfeld [10] who studied SpHb monitoring in low blood loss orthopedic surgery, and revealed that; the RBC transfusion rate was much higher (4.5\%) in the Control Group than (0.6\%) the SpHb Group, so lower rate of blood transfusion by $87 \%$ (4.5\% versus $0.6 \%$ ) and decreased mean of RBC units transfused in SpHb group than control group [10]. 
Table 3. Laboratory hemoglobin difference between both groups.

\begin{tabular}{|c|c|c|c|}
\hline & Group I & Group II & $\mathrm{P}$ value \\
\hline Baseline Hb (g/dL) & $12.2 \pm 1.7$ & $12 \pm 1.1$ & 0.02 \\
\hline Pre-transfusion $\mathrm{Hb}$ (g/dL) & $8.2 \pm 1.3$ & $8.7 \pm 1.1$ & 0.33 \\
\hline Post-transfusion $\mathrm{Hb}$ (g/dL) & $10.9 \pm 0.7$ & $10.3 \pm 0.4$ & 0.04 \\
\hline Transfusion delay time (time from determination of need to transfused) min & $43.5 \pm 8.6$ & $10.5 \pm 2.3$ & 0.001 \\
\hline
\end{tabular}

Data are described as mean $\pm \mathrm{SD}, \mathrm{P}$ value $<0.05$ is considered statistically significant.

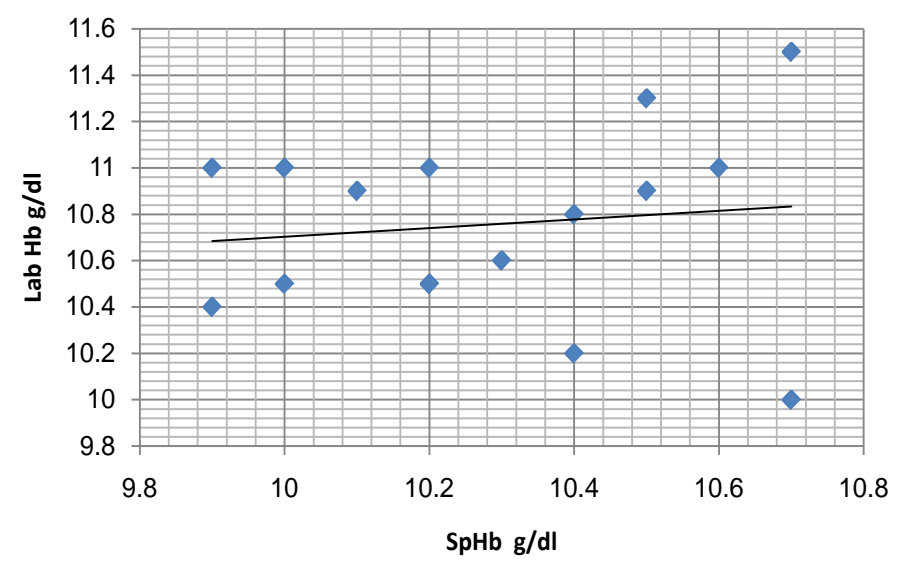

Figure 1. Correlation coefficient between lab $\mathrm{Hb}$ after transfusion and SpHb after transfusion $(r=0.045, \mathrm{p}>0.05)$.

Our results also agreed with Miller et al. [13] who compared three methods of hemoglobin monitoring in Patients undergoing spine surgery, and concluded that SpHb could have frequently been used in many patients to guide clinical decisions regarding the need for blood transfusion [13]. Wael et al. [12], who compared continuous hemoglobin monitoring versus laboratory method in patients undergoing brain tumor resection, and concluded that continuous hemoglobin monitoring affects transfusion behavior, allowing earlier cessation of RBC transfusion as well as earlier consideration of initiation of RBC transfusion [12]. Also, our results are similar to results of Causey et al. [14] who found SpHb to have clinically acceptable accuracy during most types of surgery including those with significant blood loss [14].

Pre-transfusion hemoglobin in our study were $(8.7 \pm 1.1)$ in the SpHb Group while $(8.2 \pm 1.3)$ in the Control Group ( $p$ value $>0.05$ ), we believe this occurred due to the ability of the anesthesiologist to gauge in real time that the transfusion should be initiated. Although the post-transfusion hemoglobin was significantly different between groups with the Control Group being higher ( $\mathrm{p}$ value < 0.05), SpHb allowed a faster decision to cessation a transfusion preventing further RBC units from being transfused, this is due to a delay in laboratory Hb values. Unfortunately, $\mathrm{Hb}$ lab is relatively expensive and is often time-consuming, resulting in a "snapshot” impression of changes in Hb concentration overtime. SpHb would, therefore, be a major advantage [15].

Our results also revealed that, by using Pearson correlation coefficient to detect the correlation between Lab $\mathrm{Hb}$ and $\mathrm{SpHb}$, there was a highly significant correlation between baseline Lab Hb and baseline SpHb $(r=0.698$, p < 0.001). Similarly, Lab Hb before transfusion showed a significant correlation between SpHb before transfusion $(r=0.710, p<0.001)$. On the contrary, there was a non-significant correlation between Lab Hb after transfusion and SpHb after transfusion ( $r=0.045$, $\mathrm{p}>0.05$ ). The accuracy of SpHb has been evaluated in Berkow and his colleagues [16] who evaluated absolute accuracy. While trend accuracy, which in our opinion is of greater importance when evaluating a monitoring technology. Unfortunately, laboratory analysis only offers a single point measurement, while hemoglobin is continuously changing. This result correlates well with Lacroix J. et al. [17] who described that SpHb provides a "motion picture". In our study, the ability to watch the continuous trend in hemoglobin allows earlier consideration of RBC transfusion as well as an earlier cessation of RBC transfusion. Post-operatively, continuous trending could indicate hemorrhage that is otherwise not apparent. Macknet et al. [18] who found a highly significant correlation between laboratory Hb and $\mathrm{SpHb}\left(\mathrm{R}^{2}=0.94\right)$. 
Also, this cope with Vos et al. [15] who found a significant correlation between laboratory Hb and SpHb $\left(\mathrm{R}^{2}=\right.$ 0.5) for all data. Causey et al. [14] found highly significant correlation between Masimo SpHb and laboratory $\mathrm{Hb}$ in operative room and intensive care unit groups (0.77, 0.67 respectively).

In our study, the correlation dramatically increased before transfusion (i.e. with low $\mathrm{Hb}<10 \mathrm{gm} / \mathrm{dL}$ ). This agrees with Masimo FDA submission data which revealed increased accuracy to $80 \%$ instead of $68 \%$ in $\mathrm{Hb}$ below $10 \mathrm{gm} / \mathrm{dL}$. Same results had been obtained by Vos et al. [15].

In contrast to our results Gayat et al. [19] who found that $\mathrm{SpHb}$ was less accurate. Different results can be explained by the different methodology, as the investigators were used an earlier version of the SpHb sensor in their study.

Our study has some limitations. Unfortunately, we were not able to collect post-surgery transfusion data or post-discharge information, which would enhance the value of our investigation.

\section{Conclusion}

Finally, we can conclude that noninvasive hemoglobin monitor can guide blood transfusion in cancer patients. Furthermore, it reduces rate and total units of blood.

\section{Financial Support}

No financial support was received for this work.

\section{Interest}

No conflict of interest declared.

\section{References}

[1] Acheson, A.G., Brookes, M.J. and Spahn, D.R. (2012) Effects of Allogeneic Red Blood Cell Transfusions on Clinical Outcomes in Patients Undergoing Colorectal Cancer Surgery: A Systematic Review and Meta-Analysis. Annals of Surgery, 256, 235-244. http://dx.doi.org/10.1097/SLA.0b013e31825b35d5

[2] Boehm, K., Beyer, B., Tennstedt, P., Schiffmann, J., Budaeus, L. and Haese, A. (2015) No Impact of Blood Transfusion on Oncological Outcome after Radical Prostatectomy in Patients with Prostate Cancer. World Journal of Urology, 33, 801-806. http://dx.doi.org/10.1007/s00345-014-1351-0

[3] Beattie, W.S., Karkouti, K., Wijeysundera, D.N. and Tait, G. (2009) Risk Associated with Preoperative Anemia in Noncardiac Surgery: A Single-Center Cohort Study. Anesthesiology, 110, 574-581. http://dx.doi.org/10.1097/ALN.0b013e31819878d3

[4] Shander, A., Javidroozi, M., Perelman, S., Puzio, T. and Lobel, G. (2012) From Bloodless Surgery to Patient Blood Management. Mount Sinai Journal of Medicine, 79, 56-65. http://dx.doi.org/10.1002/msj.21290

[5] Carson, J.L., Carless, P.A. and Hebert, P.C. (2012) Transfusion Thresholds and Other Strategies for Guiding Allogeneic Red Blood Cell Transfusion. Cochrane Database of Systematic Reviews, 4, 20-24. http://dx.doi.org/10.1002/14651858.cd002042.pub3

[6] Frank, S.M., Savage, W.J., Rothschild, J.A., Rivers, R.J., Ness, P.M., Paul, S.L. and Ulatowski, J.A. (2012) Variability in Blood and Blood Component Utilization as Assessed by an Anesthesia Information Management System. Anesthesiology, 117, 99-106. http://dx.doi.org/10.1097/ALN.0b013e318255e550

[7] Seruya, M., Oh, A.K., Boyajian, M.J., Myseros, J.S., Yaun, A.L. and Keating, R.F. (2011) Unreliability of Intraoperative Estimated Blood Loss in Extended Sagittal Synostectomies. Journal of Neurosurgery: Pediatrics, 8, 443-449. http://dx.doi.org/10.3171/2011.8.peds11180

[8] Goldman, J.M., Petterson, M.T., Kopotic, R.J. and Barker, S.J. (2000) Masimo Signal Extraction Pulse Oximetry. Journal of Clinical Monitoring and Computing, 16, 475-483. http://dx.doi.org/10.1023/A:1011493521730

[9] Knutson, C., Della-Giustina, C., Tomich, C., Wills, B. and Leurssen, C. (2010) Does a Portable, Noninvasive Hemoglobin Monitor Effectively Correlate With Venous Blood Levels? Annals of Emergency Medicine, 56, S133. http://dx.doi.org/10.1016/j.annemergmed.2010.06.535

[10] Ehrenfeld, J.M., Justin, P., Catherine, M. andWarren, S. (2014) Impact of Continuous and Noninvasive Hemoglobin Monitoring on Intraoperative Blood Transfusions. Journal of Blood Disorders, 5, 9.

[11] Frasca, D., Mounios, H., Giraud, B., Boisson, M., Debaene, B. and Mimoz, O. (2015) Continuous Monitoring of Haemoglobin Concentration after In-Vivo Adjustment in Patients Undergoing Surgery with Blood Loss. Anaesthesia. 
[12] Wael, N., Maher, F., Mohmoued, M., Radwan, T., Gomaa, Z. and Hany, W. (2015) Continuous and Noninvasive Hemoglobin Monitoring Reduces Red Blood Cell Transfusion during Neurosurgery. Journal of Clinical Monitoring and Computing, 29, 733-740. http://dx.doi.org/10.1007/s10877-015-9660-4

[13] Miller, R.D., Ward, T.A., Shiboski, S.C. and Cohen, N.H.A. (2011) Comparison of Three Methods of Hemoglobin Monitoring in Patients Undergoing Spine Surgery. Anesthesia \& Analgesia, 112, 858-863. http://dx.doi.org/10.1213/ANE.0b013e31820eecd1

[14] Causey, M.W., Miller, S., Foster, A., Beekley, A., Zenger, D. and Martin, M. (2011) Validation of Noninvasive Hemoglobin Measurements Using the Masimo Radical-7 SpHb Station. The American Journal of Surgery, 201, 590-596. http://dx.doi.org/10.1016/j.amjsurg.2011.01.020

[15] Vos, J.J., Kalmar, A.F., Struys, M.M.R.F., Porte, R.J., Wietasch, J.K.G., Scheeren, T.W.L. and Hendriks, H.G.D. (2012) Accuracy of Non-Invasive Measurement of Haemoglobin Concentration by Pulse Co-Oximetry during SteadyState and Dynamic Conditions in Liver Surgery. British Journal of Anaesthesia, 109, 522-528. http://dx.doi.org/10.1093/bja/aes234

[16] Berkow, L., Rotolo, S. and Mirski, E. (2011) Continuous Noninvasive Hemoglobin Monitoring during Complex Spine Surgery. Anesthesia \& Analgesia, 113, 1396-1402. http://dx.doi.org/10.1213/ANE.0b013e318230b425

[17] Lacroix, J. and Tucci, M. (2012) Noninvasive or Invasive Hemoglobin Measurement? Critical Care Medicine, 40, 2715-2716. http://dx.doi.org/10.1097/CCM.0b013e31825bc975

[18] Macknet, M.R., Allard, M., Applegate, R.L. and Rook, J. (2010) The Accuracy of Noninvasive and Continuous Total Hemoglobin Measurement by Pulse Co-Oximetry in Human Subjects Undergoing Hemodilution. Anesthesia \& Analgesia, 111, 1424-1426. http://dx.doi.org/10.1213/ANE.0b013e3181fc74b9

[19] Gayat, E., Antoine, B., Caroline, S., Mereille, B. and Marc, F. (2011) Performance Evaluation of a Noninvasive Hemoblobine Monitoring Device. Annals of Emergency Medicine, 58, 106-107. 